\title{
Compacta: a fast contig clustering tool for de novo assembled transcriptomes
}

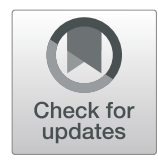

Fernando G. Razo-Mendivil ${ }^{1}$, Octavio Martínez ${ }^{2^{*}}$ and Corina Hayano-Kanashiro ${ }^{\text {* }^{*}}$ (1)

\begin{abstract}
Background: RNA-Seq is the preferred method to explore transcriptomes and to estimate differential gene expression. When an organism has a well-characterized and annotated genome, reads obtained from RNA-Seq experiments can be directly mapped to that genome to estimate the number of transcripts present and relative expression levels of these transcripts. However, for unknown genomes, de novo assembly of RNA-Seq reads must be performed to generate a set of contigs that represents the transcriptome. These contig sets contain multiple transcripts, including immature mRNAs, spliced transcripts and allele variants, as well as products of close paralogs or gene families that can be difficult to distinguish. Thus, tools are needed to select a set of less redundant contigs to represent the transcriptome for downstream analyses. Here we describe the development of Compacta to produce contig sets from de novo assemblies.

Results: Compacta is a fast and flexible computational tool that allows selection of a representative set of contigs from de novo assemblies. Using a graph-based algorithm, Compacta groups contigs into clusters based on the proportion of shared reads. The user can determine the minimum coverage of the contigs to be clustered, as well as a threshold for the proportion of shared reads in the clustered contigs, thus providing a dynamic range of transcriptome compression that can be adapted according to experimental aims. We compared the performance of Compacta against state of the art clustering algorithms on assemblies from Arabidopsis, mouse and mango, and found that Compacta yielded more rapid results and had competitive precision and recall ratios. We describe and demonstrate a pipeline to tailor Compacta parameters to specific experimental aims.
\end{abstract}

Conclusions: Compacta is a fast and flexible algorithm for the determination of optimum contig sets that represent the transcriptome for downstream analyses.

Keywords: RNA-Seq, de novo assembly, Corset, Grouper, Transcriptomics

\section{Background}

RNA-Seq is the most frequently used method to explore transcriptomes, i.e., sets of mRNA molecules expressed in a cell, tissue, organ or whole organism under particular conditions $[1,2]$. To generate samples for RNA-Seq, mRNA isolated from a given sample is converted to circular DNA (cDNA) that includes a mixture of fragments. The cDNA is sequenced to obtain 'reads' that represent parts of the original mRNA molecules. When a sample genome is known, the reads can be mapped to

\footnotetext{
*Correspondence: octavio.martinez@cinvestav.mx; angela.hayano@unison.mx ¿Unidad de Genómica Avanzada (Langebio), Centro de Investigacíon y de Estudios Avanzados del Instituto Politécnico Nacional (Cinvestav), Irapuato, Gto, Mexico

${ }^{1}$ Departamento de Investigaciones Científicas y Tecnológicas de la

Universidad de Sonora, Universidad de Sonora, Hermosillo, Mexico
}

a reference sequence to reconstruct the transcripts and estimate their relative abundance.

However, when no genome is available, reads must be assembled de novo before attempting to reconstruct the expressed transcripts and estimate their relative abundance. Transcriptome assemblers including Trinity [3], Soap de novo [4], ABySS [5] or Spades [6], among others, perform this assembly to generate 'contigs' - sequences arising from reads that overlap or by the use of 'Brujin graphs' [7].

De novo assembly of eukaryotic transcriptomes is challenging both due to dataset size that can include billions of reads and the difficulties in identifying alternatively spliced variants [7], alternative gene alleles [8], small variants within a gene family [5] or close gene paralogs $[9,10]$. This assembly problem is exacerbated

(C) The Author(s). 2020 Open Access This article is distributed under the terms of the Creative Commons Attribution 4.0 International License (http://creativecommons.org/licenses/by/4.0/), which permits unrestricted use, distribution, and 
by temporal transcription, wherein significant parts of the genome, both coding and non-coding segments, are transcribed only at specific points during development or under specific conditions [11, 12]. Moreover, a large fraction of reads can belong to nascent RNAs, and thus include introns that could contribute to many contigs in the assembly [13]. As a result, transcriptome assemblies typically produce very large contig sets that in some cases are many-fold larger than the number of genes in the entire species genome. For example, de novo assembly of the transcriptome for the polychaete annelid Platynereis dumerilii using Trinity gave a set of 273,087 non-redundant contigs, which were identified through a pipeline that included sequence homology to only 17 , 213 genes [14], nearly 16-fold fewer than the number of contigs.

Transcriptome assemblers output many contigs that reflect the diversity found in the original mixture of mRNA molecules. However, for downstream analyses, these large contig collections must be culled to yield a smaller and more tractable set, which ideally groups contigs into transcripts produced by the same gene. Methods to group contigs can involve the use of sequence information, such as cd-hit-est [15], or use only the information about which reads map to each contig. The two main programs using the second approach are Corset [16] and Grouper [17].

Corset takes the set of reads and hierarchically clusters the contigs based on the proportion of shared reads. The program first filters out contigs that have a low number of mapped reads ( $<10$ by default) and then cluster contigs based on shared reads, while separating contigs having different expression patterns between samples. This approach thus avoids placing two or more paralogs or alternatively spliced forms into the same cluster through the use of a likelihood ratio test across groups of samples having a fixed $P$ value threshold of approximately $10^{-5}$. A distance threshold for clustering can be set by the user, but the default value of 0.3 is equivalent to sharing of $70 \%$ of the reads between two entities, i.e., original contigs or clusters already obtained by the algorithm. The number of shared reads is also updated at each iteration and clustering of a contig set stops when either all the contigs have been grouped into a single cluster or the current minimum distance increases above the distance threshold.

The Corset algorithm has two disadvantages: First, it uses a fixed number of reads to assess contig coverage, disregarding contig and read lengths; Second, and perhaps more importantly, the Corset algorithm depends heavily on results of a likelihood ratio test to segregate into clusters those contigs that could be the product of two different genes. The nature and number of conditions used to obtain different transcriptome samples can be unpredictable and, in principle, extremely diverse. However, Corset output depends on these conditions and thus groups working with the same organism could conceivably obtain significantly different sets of clusters to represent the transcriptome. Also, for annotation of ongoing eukaryotic genome projects, an equimolar mixture of RNA from different tissues of the same species is sequenced [18]; in these cases the approach used by Corset that segregates contigs from the same gene is not useful because only one 'condition' is used and thus a maximum likelihood test cannot be performed.

Grouper is another algorithm that generates contig clusters based on shared reads. Similar to Corset, outputs generated by the Grouper algorithm exclude contigs having fewer than 10 reads; this threshold cannot be modified by the user. Also, like Corset, Grouper uses a likelihood ratio test of expression estimates that vary significantly across conditions to separate contigs under the assumption that such contigs arose from different paralogous genes. Optional Grouper filters allow information for 'orphan' reads (when paired reads are used), whereas the 'min-cut' filter uses the likelihood ratio test to completely separate contigs, thus avoiding long path joining. Interestingly, Grouper does not have a user adjustable threshold for weight (or distance) by which contigs are clustered and instead relies only on the abovementioned filters to cluster or segregate contigs. Grouper also has an associated module to label (annotate) clusters using information from a closely related genome.

Grouper shares the same disadvantages with Corset, i.e., the program uses an arbitrary minimum number of reads to consider whether a contig is valid (in Grouper the user cannot modify this value) and contig segregation depends on the RNA-Seq experimental conditions.

The ideal behavior for an algorithm to cluster contigs obtained by de novo assembly of a transcriptome would be to output a group of clusters (contig sets) that perfectly represent actual gene expression, i.e., a set wherein the relationship between cluster and gene is one to one. There are strong arguments concerning the impossibility of obtaining such an 'ideal' algorithm in the absence of detailed knowledge about the genome sequence in question and using only the information given by multimapping files that relate reads to contigs. In mathematical terms, we have an identifiability problem, meaning that different sets of parameters (genes) can give a set of reads having identical statistical profiles (number of reads per contigs), making it impossible to determine the set of genes that generated the output. As clearly demonstrated by [19], to correctly identify transcripts based entirely on RNA-Seq data, at minimum geneboundary data are needed, and data concerning transcription start sites, splice junctions and polyadenylation 
sites are also useful. As noted by Boley et al. [19], "This means that it is not always possible to positively identify alternative transcript isoforms, even as the read depth approaches infinity". Confronted with the problem of clustering contigs from an unknown genome, we have no information concerning factors such as genome size and complexity [20,21], allele and gene copy variations [22] or variations in exon-intron architecture [23]. Under this scenario, the best use of information from de novo assembly is formation of a contig cluster that can be used to identify the core set of expressed genes that allows the most effective comparison of the relative expression of such entities based on the design of the RNA-Seq experiment.

With the aim of reducing the complexity of RNA-Seq data analyses, we present Compacta, a fast, flexible, and computationally efficient way to group contigs obtained from de novo assembly into clusters to represent the core set of genes expressed in a given experiment as well as to allow identification of gene sets and enhance statistical power for detection of differential expression. The algorithm depends on only two parameters: filtering of low coverage contigs based on effective coverage and clustering strength. After running Compacta, a single contig, representing each cluster obtained, can be used for downstream analyses for gene identification and detection of differential gene expression.

\section{Implementation}

Compacta is designed to reduce the number of contigs to a smaller set of representative sequences while preserving the information about relative expression given by read abundance. Its output can be used for downstream analyses to identify contigs and differential gene expression patterns.

Prior to using Compacta, transcriptomes must be assembled de novo using tools such as Trinity [3], Soap de novo [4] or Spades [6]. Sequencing reads are then mapped back to the assembled transcriptome using alignment-based software such as Bowtie2 [24] or Hisat2 [25] to obtain a multi-mapped binary file in the 'BAM' format [26]. BAM files are the initial input for Compacta and contain information about the contig set given by the assembler as well as the reads that map to each set.

Compacta has two core parameters, $-\mathrm{d}=d$, a threshold for when two contigs belong to the same cluster, and $-\mathrm{l}=l$, the threshold needed for the minimum effective coverage for a contig to enter the clustering algorithm. The value for $d$ ranges between zero and one and controls the extent of clustering. When $d=0.3$, for example, all pairs of contigs sharing $30 \%$ or more of the reads that reference the contig having fewer reads will be clustered into a single entity. Meanwhile, $l=2$ implies that only those contigs having a total coverage that is twice the contig length in terms of sequencing read lengths will enter into the clustering process. Default values for these two parameters are $d=0.3$ and $l=2$, which are determined in the input as "-d $0.3-12$ ". In addition to file locations, Compacta includes options for number and names of samples and experimental groups, as well as options that allow parallelization of part of the algorithm.

Compacta output comprises files that: (i) define the obtained clusters as sets of the original contigs; (ii) give the number of reads (raw count) of each cluster for each sample input; and (iii) describe the type of clusters obtained. The following list describes the parameters of the Compacta algorithm.

1. Input. A set of BAM files and Compacta options. BAM file data are parsed for the next step. The sample origin of reads is preserved for inclusion in the output.

2. Graph computation. From sets of $c$ contigs and $r$ reads in BAM files, Compacta creates an undirected graph with $c$ vertices corresponding to contigs and $c(c-1) / 2$ connections (edges) between vertices. The weight, $w_{i j}$, of an edge connecting contigs $i$ and $j ; i$ $-j$, is calculated

$$
w_{i j}=\frac{R_{i j}}{\min \left(R_{j}\right)}
$$

where $R_{i}$ and $R_{j}$ are the number of reads that independently map to contigs $i$ and $j$, respectively, while $R_{i j}$ is the total number of reads that map to both contigs $i$ and $j$; i.e., $R_{i j}$ is the number of reads shared by contigs $i$ and $j$. This function is well defined since $\min \left(R_{i} R_{j}\right)>0$. The weight of an edge, $w_{i j}$, ranges from zero, when the edge contigs share no sequencing reads indicating no similarity (disconnected contigs), to one, indicating that one of the contigs is a proper subset of the other.

1. Filtering of low evidence contigs. The value $c_{i}$ is defined as the length of contig $i$ and $s_{i}$ is the sum of the lengths of all reads that map to that contig. If $s_{i}<\left(l \times c_{i}\right)$, where $l$ is the parameter ' -1 ' input by the user, the contig $i$ is disconnected from any other vertices in the graph and will be reported as a low evidence contig'. Disconnection of contig $i$ implies setting all weights $w_{i j}=0$ for all values of $j$, in turn implying that when the set of contigs considered in subsequent algorithm steps fulfill the condition $s_{i} \geq\left(l \times c_{i}\right)$, they are considered to be contigs with sufficient evidence of expression.

2. Pre-cluster detection. Connected contigs (vertices) are detected and isolated sub-graphs are 
marked as 'pre-clusters' that are each loaded into a heap structure self-ordered by edge weight, ensuring that the first value in the heap is always the edge having the heaviest weight, i.e., the largest value of $w_{i j}$.

3. Clustering. Compacta processes each pre-cluster using an agglomerative algorithm. At each iteration, the algorithm selects the edge having the highest weight and, if this weight is above the defined threshold $d$ (parameter input as $-\mathrm{d}$ ), the nodes are grouped into a new entity. In this scenario, weights, $w_{i j}$, are re-calculated for the new conformation of the pre-cluster and the process is repeated until the first edge in the heap has a weight that is less than the threshold $d$ or all its contigs are clustered together. The final content of the heap structure, which can contain one or more clusters, goes to the output.

4. Output. Once Compacta processes all pre-clusters, it produces files that include the description of each cluster (sets of the original contigs), as well as lists indicating which contig could represent each one of the clusters, either by being the longest contig in the cluster or the one that has the largest number of reads mapping to it.

In summary, from BAM files containing the information of the original contigs and reads mapping to them, Compacta produces a set of representative contigs for use in downstream analyses.

\section{Algorithm implications}

As with other software designed to reduce transcriptome complexity, such as Corset or Grouper, Compacta uses a graphical approach that ignores nucleotide sequence and considers contigs only as sets of sequencing reads. Two contigs, $i$ and $j$, will be connected in the graph if they share some reads, i.e., if their intersection is not empty and $w_{i j}>0$. In step (2) of the algorithm, the graph is constructed. Even when in principle all pair comparisons between contigs must be performed, only the ones for which the weights are larger than zero $\left(w_{i j}>0\right)$ need to be stored and analyzed downstream. The logic behind weight calculation is that contigs sharing a large proportion of reads will also be 'alike' at the sequence level, allowing read position within contigs to be disregarded. Thus, if $w_{i j}=0$ we will consider that the corresponding contigs are completely unrelated, whereas $w_{i j}=1$ means that the smaller contig is a proper subset of the second, or, when they are the same size, they will be some permutation of the positions of the same reads.

In step (3) of the algorithm, Compacta uses effective contig coverage, expressed as the number of times that the full-length contig is covered by reads, as a measure to detect and discard low evidence contigs. The user controls the strength of filtering via parameter $l$; By setting $l=3$, for example, only those contigs having sufficient numbers of reads to cover the contig length three times will pass the filter and continue for downstream analysis. This parameter allows the user to limit the subset of contigs of interest. Thus, if only those genes having high expression levels are relevant, $l$ can be set to a high value. Filtered contigs are not discarded, but are included in the output in which they are identified as 'low evidence singletons'. In contrast, Corset and Grouper allow selection of contigs only through a fixed threshold in the number of reads that map to each contig, independently of contig length. In Corset this threshold can be changed by the user and by default is set to 10 , while in Grouper the threshold is fixed as 10 reads. However, a fixed threshold number of reads is inadequate to judge contigs having different lengths. For example, consider the situation in which reads of $250 \mathrm{bp}$ are used and a contig of length $750 \mathrm{bp}$ is produced by 9 overlapping reads. Here, the effective contig coverage is $(250 \times 9) /$ $750=3$, and Compacta will reasonably pass such a highly covered contig for any value of $l \leq 3$, whereas Corset and Grouper would discard such a contig considering it as 'low coverage', and thus it would not appear in the output.

The graph constituted by all contig pairs having $w_{i j}>0$ are input into the fourth step of the algorithm, 'pre-cluster detection'. Here a pre-cluster is defined as a set of inter-connected contigs, or, in graph theory terms, as a 'connected graph' [27]. In simple terms, in a pre-cluster there is a path that connects, either directly or indirectly, all contigs that form such a structure. If a pre-cluster graph is plotted, it is possible to go from any of the contigs to any other contig by following a path. An important computational advantage of Compacta is that each pre-cluster is loaded into a self-ordered heap structure, in which the first edge always has the largest $w_{i j}$ value. This heap structure is similar to ordered binary trees, and can save considerable time [28], because arrays having millions of components are not sorted at each iteration.

The core of the Compacta algorithm is step (5), involving agglomerative clustering of connected contigs or 'pre-clusters' that can be performed in parallel. The processing of each pre-cluster is independent of other data, and thus its clustering can be sent as an independent thread, making optimal use of computer resources. With the same goal, sets of pre-clusters could be distributed to independent nodes in computer clusters. Clustering of a pre-cluster structure proceeds by grouping into a single entity pairs of sets having weight $w_{i j}$ that surpass the threshold $d$ input by the user. Given that the precluster is loaded into a self-ordered heap, the algorithm 
needs only to analyze the first element of the heap, thus saving valuable time. Clustering of two entities, $i$ and $j$ (that could be original contigs or previously identified clusters), happens only if $w_{i j} \geq d$ and in that case both entities are grouped together, after which weights between the new entity and all those in the pre-cluster are re-calculated and the algorithm iterated. In the opposite case, such as when $w_{i j}<d$ during the iterations, the entire content of the heap is sent to the output, including the definitions of clusters and the number of reads that map to them. This process guarantees that the number of entities in the output is smaller than or at most equal to the number of input contigs. A simple example of this process is presented in Section 1 of Additional file 1.

Any contig clustering algorithm that does not use direct sequence information but instead uses a graphical approach must have a parameter homolog to the weight threshold $d$ used by Compacta. For example, in Corset and Grouper this homolog parameter is the distance between contigs, which is simply the inverse additive of Compacta $d$, i.e., $1-d$ for the threshold and $1-w_{i j}$ for the weights, which in these programs are conceptualized as distances. In addition to the criterion used to filter 'low evidence contigs' as mentioned earlier, computational implementation of Compacta differs from those in Corset and Grouper in the use of efficient self-sorting heap structures to dynamically store pre-clusters, which in turn allows the clustering step of Compacta to be fully parallelized or distributed, thus making optimum use of computer resources, including multi-core clusters.

Another substantial way that Compacta differs from Corset and Grouper is that Compacta uses no computational methods to determine if two contigs were the product of transcription from 'the same gene', whereas both Corset and Grouper attempt to estimate and consider contig origin. In our opinion, in the absence of genomic information, accurate prediction of whether two contigs are the product of: a) different alleles of the same gene, b) alternative splicing forms produced from the same gene or c) two highly similar genes (close paralogs or two close members of the same gene family) is essentially impossible due to the high diversity and conformations of eukaryotic genomes.

Compacta will be particularly useful when no genome is available for a given organism, and the researcher wants to: a) Have a core set of sequences representing the major expressed genes that allows putative identification via comparisons with well-known orthologs; and b) Perform differential expression analysis of core genes expressed in the transcriptome. To achieve these aims, the ability to downsize the potentially very large number of contigs given by the assembler into a smaller and more manageable set of representative sequences is valuable.

\section{Adjusting Compacta to assembly complexity}

RNA-Seq experiments capture many transcript types such as nascent or pre-mature RNAs [13] or non-coding sequences like long non-coding RNAs [29]. In fact, the ratio of transcribed non-coding to coding sequences can vary enormously; in humans this ratio is $47: 1$, but in nematodes is only 1.3:1 [30]. The assembly process is likely to yield many related contigs that represent transcription variants of the same gene as alternative splicing forms, alleles, or products of the transcription of close paralogs of the same gene or gene family. Here we discuss the features that Compacta offers to reduce assembly complexity in a general framework.

Given a particular assembly, say $\mathbf{t}$, consisting of a group of $c$ contigs and $r$ reads related by multi-mapping files ('BAM' files), we can use Compacta to reduce the set of c contigs to a smaller set of $z$ representative clusters such that $z \leq c$. Apart from filtering low-evidence contigs with the parameter $-1=l$, the number of clusters given by the algorithm is a function only of the parameter $d$-the threshold for clustering contigs into clusters, say $f(\mathbf{t}, d)=z$, or simply $f(c, d)=z$, considering only the number of input contigs, $c$, and the number of clusters output, $z$. By setting $d=0$ we will cluster all contigs that share one or more reads, because in that case all contig pairs $\{i, j\}$ that fulfill $R_{i j}>0$ will give a weight $w_{i j}>0$ and thus be clustered together, giving the smallest number of clusters in the output. The number of clusters resulting from that operation can be termed $z_{\text {min }}$, where $f(c$, $d=0)=z_{\text {min }}$, which represents the maximum assembly reduction that can be achieved by the algorithm. By clustering all contigs with the slightest evidence of sequence similarity (i.e., one or more shared reads) we can group all alleles, alternative splicing variants and close paralogs genes into a single cluster. However, using this approach we could also group into a single cluster transcripts produced by different genes that share sequence motifs that expand in sequence length beyond the length of a single read. Under the same experimental conditions, and with high sequencing depth, we can assume that read length will have a strong effect in determining the value of $z_{\text {min }}$; short reads will cause $z_{\text {min }}$ to be smaller than when long reads are used. On the other hand, if $d$ is set to 1 , we will ask the algorithm to group only contigs that share all reads of the smaller contig, because in order to have $w_{i j}=R_{i j} / \min \left(R_{i j} R_{j}\right)=1$ we must have $R_{i j}=R_{i}$ or $R_{i j}=R_{j}$. In that case, we will have a maximum number of clusters in the output, where $f(c, d=$ $1)=z_{\max }$, such that Compacta will cluster only those contigs that are proper subsets of the longest contig in the group (pre-cluster) and will likely produce clusters containing only highly similar gene alleles, splicing forms that share most exons in the genes, or very close paralogs. Taken together, from this analysis we can conclude 
that $f(c, d)=z$ is a non-decreasing function of $d$ with domain in the interval $[0,1]$ for $d$ and co-domain in $\left[z_{\text {min }}\right.$, $z_{\text {max }}$ ] for $z$. The fact that $f(c, d)=z$ is non-decreasing follows from the fact that a larger value of $d$ can only increase the number of output clusters, $z$, given that the clustering algorithm will be more stringent, i.e., if $d_{1}<$ $d_{2}$ then $f\left(c, d_{1}\right) \leq f\left(c, d_{2}\right)$. Due to the speed of Compacta, performing two runs with extreme values, $d=0$ and $d=$ 1 , to obtain the values of $z_{\text {min }}$ and $z_{\max }$ for a particular assembly is not computationally expensive. Having the range of possible $z$ values allows the researcher to fix a target value $z^{*}, z_{\text {min }} \leq z^{*} \leq z_{\text {max }}$, and, using a numerical method, obtain the value of $d$ (e.g., $\left.d^{*}\right)$, such that $f(c$, $\left.d^{*}\right) \approx z^{*}$ by performing a set of Compacta runs.

\section{Source data and software evaluation}

Three RNA-Seq datasets from Arabidopsis (Arabidopsis thaliana), mango (Mangifera indica) and mouse (Mus musculus) were processed to compare Compacta with other clustering tools.

In Table 1 the 'Source' column provides the reference for the corresponding dataset; the column 'Accession' shows accession identifiers for data deposited in the Sequence Read Archive [34] of GenBank; the column 'Reads (Gb)' indicates the approximate giga base pairs of raw data; and 'Contigs' shows the number of contigs obtained from the assembly. The Arabidopsis and mouse datasets were assembled de novo using the Trinity assembler version 2.4.0 with default parameters, whereas the mango dataset assembly generated by Trinity was kindly provided by Dr. Miguel A. Hernández Oñate [32].

Compacta, Corset and Grouper were run with default parameters using as input the contigs for each assembly obtained from the sources shown in Table 1 (Fig. 1).

Results shown in Fig. 2 were obtained using Arabidopsis assembly contigs (see Table 1) and performing repeated runs of Compacta using different values of the $d$ parameter, whereas all contigs from such assemblies were identified by comparing those sequences using stringent BLAST parameters [35] with the set of all possible Arabidopsis transcripts. Details of this analysis are given in Section 3 of Additional file 1.

Results presented in Fig. 3 were obtained by running CD-HIT, Compacta, Corset, Grouper and the clustering facility of the Trinity suite on the contigs from assemblies of the Arabidopsis and mouse datasets (Table 1);

Table 1 Data sources. Sources and characteristics of the RNASeq data used in this study

\begin{tabular}{lllll}
\hline Organism & Source & Accession & Reads (Gb) & Contigs \\
\hline Arabidopsis & {$[31]$} & ERP016911 & 36.0 & 106,895 \\
Mango & {$[32]$} & SRP043494 & 62.5 & 107,744 \\
Mouse & {$[33]$} & PRJNA474181 & 41.0 & 327,616 \\
\hline
\end{tabular}

details of these experiments as well as additional analyses are given in Sections 2 and 3 of Additional file 1.

\section{Results and discussion \\ Compacta is faster than clustering alternatives}

To evaluate the absolute and relative execution time for Compacta, Corset and Grouper we used three transcriptomes from Arabidopsis, mango (Mangifera indica) and mouse (Mus musculus) assembled de novo that included $106,895,107,744$ and 327,616 contigs, respectively. All three algorithms were run with default parameters and the run time for each program with each assembly was obtained (Fig. 1; see Material and Methods for details). Table 2 shows the number of clusters output by Compacta, Corset and Grouper for the Arabidopsis, mouse and mango datasets. Compacta produced a larger number of contigs in the Arabidopsis and mouse real datasets, and the smaller number of contigs for the mango dataset and the simulated datasets of Arabidopsis and mouse. This reflects the fact that Corset and Grouper do not include contigs with low coverage in their output, while Compacta includes contigs with low coverage as single contig clusters.

In Fig. 1 the bar height corresponds to the run time for each program (bar group; $X$-axis) operating on the three assemblies that are denoted by different colors. The numbers above the bars for "Corset" and "Grouper" groups give the time taken by the program divided by the time taken by Compacta to analyze the same assembly. For example, the number 28 above the red bar for the "Corset" group indicates that Corset took approximately 28 -fold more time to finish the run for the Arabidopsis assembly than Compacta $(26.6186 h / 0.9675 h \approx$ 28).

Compacta was approximately 28-, 25- and 197-fold faster than Corset for the Arabidopsis, mango and mouse assemblies, respectively. The differences in execution time could be attributed to two factors: First, Corset uses a statistical formula to try to evaluate the gene of origin for each contig and Compacta does not; and Second, Compacta uses auto-sorting heaps, whereas Corset sorts all remaining contigs pairs in each iteration. A basic agglomerative clustering algorithm, such as that implemented for Corset, has a computation time of $O\left(n^{3}\right)$ and slows as the input size increases, as demonstrated by [28]. As mentioned above, Compacta uses an agglomerative algorithm with a heap that auto-sorts elements upon insertion and deletion that reduces computation time up to $O\left(n^{2} \log n\right)$ [28], which is considerably faster than the other algorithms, particularly when the size of the input data increases. Although Compacta may not always be faster than Corset for all possible assemblies, we predict that Compacta will be at least 10 times faster than Corset for any complex assembly from eukaryotic 

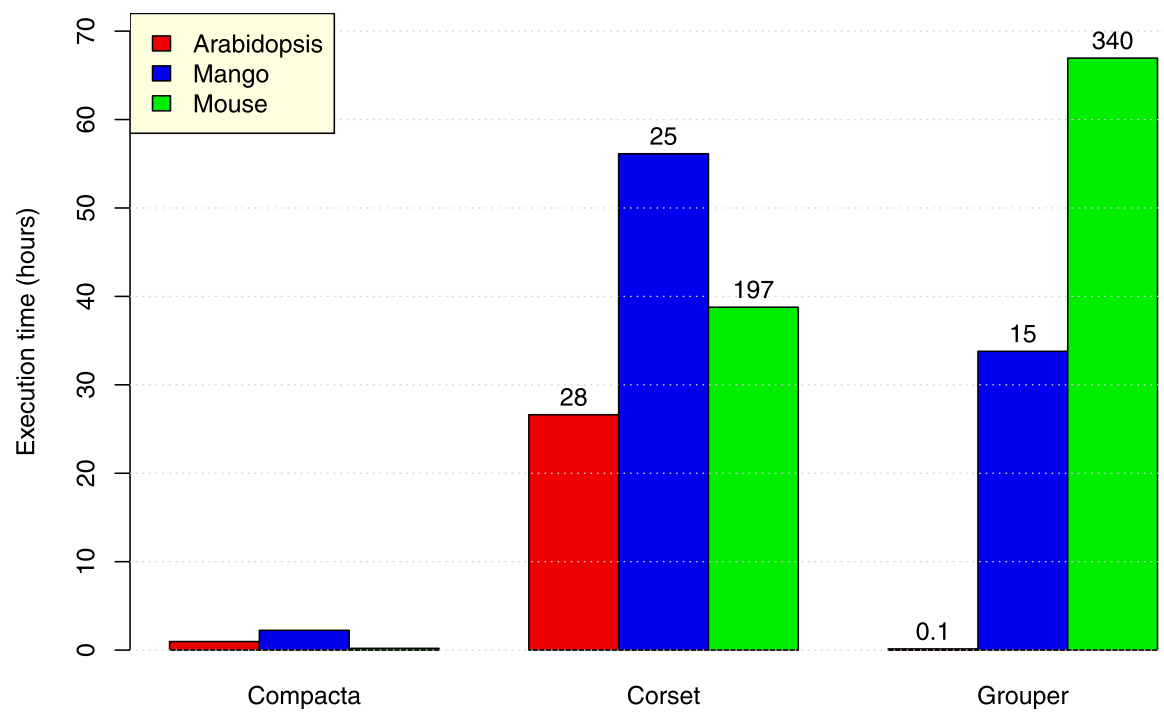

Fig. 1 Execution time for Compacta, Corset and Grouper in three assemblies. Bar diagram of running time in hours for Compacta, Corset and Grouper algorithms to analyze assemblies from Arabidopsis, mango and mouse. Numbers in the upper bars for Corset and Grouper are the number of rounds that the execution took for the corresponding program compared with the Compacta execution time

organisms. This prediction is based not only on our experimental results (Fig. 1), but also in the fundamentally more efficient way in which Compacta handles contig clustering by avoiding sorting the pre-cluster structure at each iteration, which adds significantly to the Corset run time.

On the other hand, in comparing Grouper and Compacta we see that Compacta is faster than Grouper for the mango and mouse assemblies by 15 - and 340-fold, respectively, but slower for the Arabidopsis assembly for which Compacta took $0.9675 \mathrm{~h}$ and Grouper took only
$0.1332 \mathrm{~h}$, a ratio of $\approx 0.1 \mathrm{in}$ favor of Grouper. The difference seen between Grouper and Compacta in processing the Arabidopsis assembly is due to Grouper's use of equivalence files, which are simpler to parse and contain less information than the BAM files used by Compacta. However, for larger and more complex assemblies, such as those for mango and mouse, input file parsing represents a much small fraction of the total processing time, such that Compacta is faster than Grouper (c.f., Compacta was 340-fold faster than Grouper for the mouse assembly; last bar in Fig. 1). Moreover, Grouper relies on

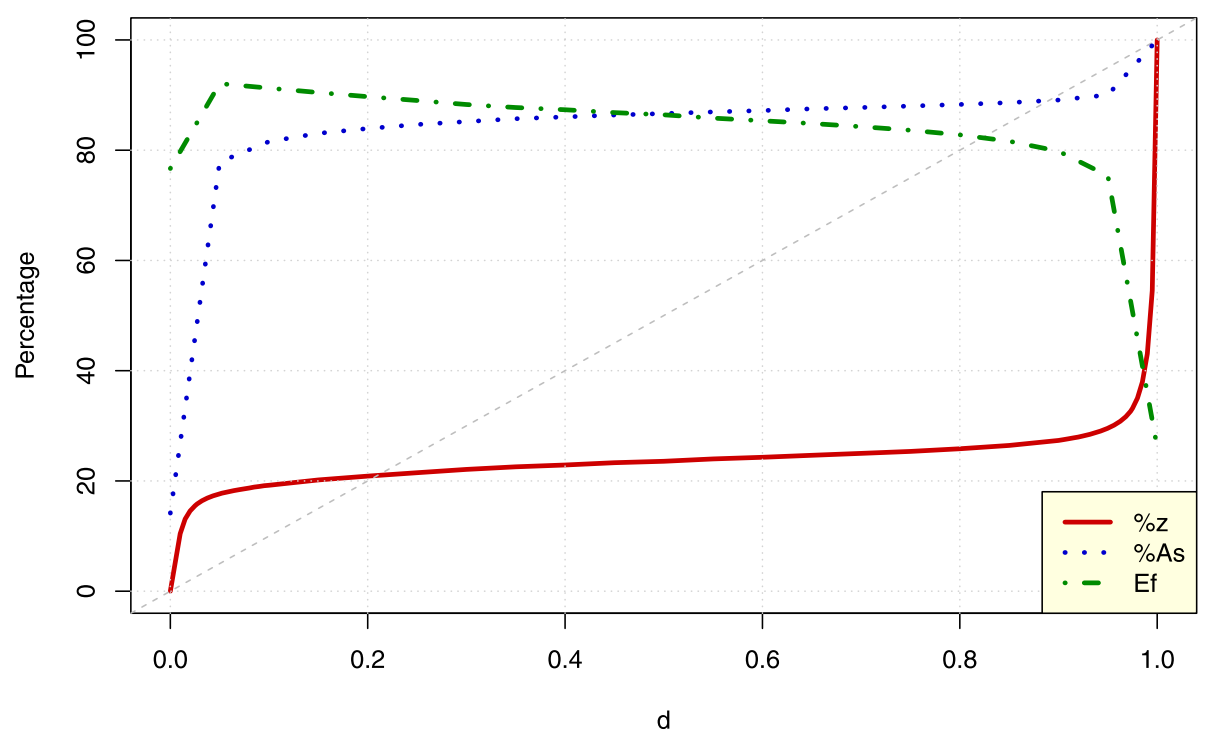

Fig. 2 Compacta results for the Arabidopsis assembly. Values for $d$ are displayed on the $X$-axis and the $Y$-axis shows the percentage of clusters ( $z$; red line), number of Arabidopsis sequences identified ( $n_{A s}$ blue dotted line) and efficiency ( $E f=n_{A s} / z$; green dashed line) as a function of $d$ 


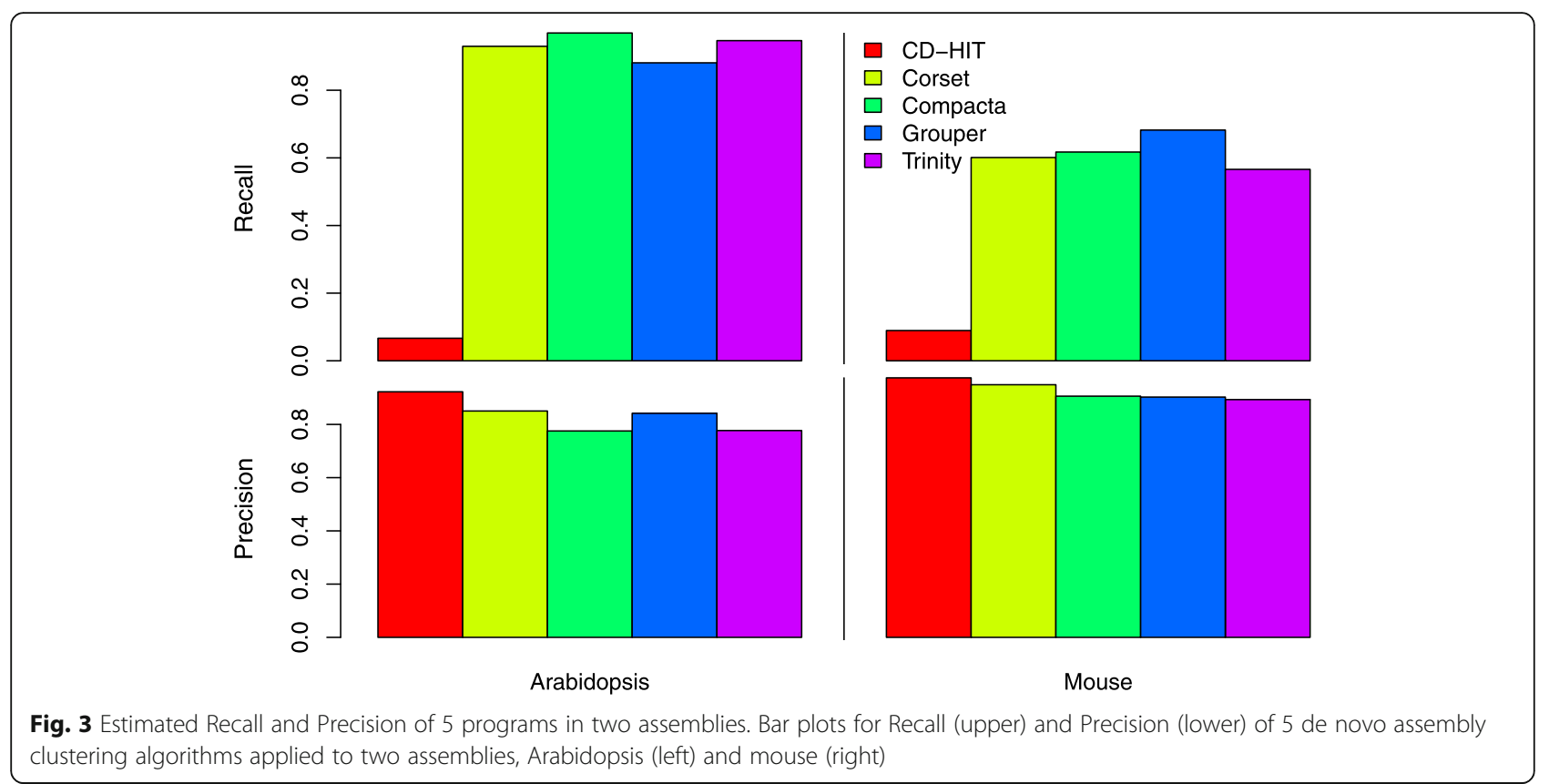

a Stoer-Wagner min-cut function that is repeatedly executed with a computation time of $O\left(m n+n^{2} \log n\right)$ [36], whereas Compacta uses the auto-sorting heap that has an execution time of $O\left(n^{2} \log n\right)$ [28]. Conservatively, Compacta is at least 10-fold faster than Grouper for clustering complex eukaryotic assemblies.

\section{Compacta adaptability to RNA-Seq objectives}

As shown above, Compacta can be adjusted through the $d$ parameter to give a number of clusters within the range $\left[z_{\text {min }} z_{\text {max }}\right]$, where $z_{\text {min }}$ corresponds to $d=0$ and $z_{\text {max }}$ corresponds to $d=1$. Here we demonstrate and discuss the consequences of selecting particular values for $d$ to adjust the clustering results according to the particular aims of an RNA-Seq experiment. Because Compacta is fast, runs can be performed for a grid of $d$ values and the results can be used for downstream analyses to identify genes and detect differential expression. Compared with the costs of RNA-Seq library construction and sequencing, the costs for bioinformatic analyses are negligible such that more time can be spent to

Table 2 Number of representative contigs selected by each algorithm from each transcriptome when run with default parameters

\begin{tabular}{llllllll}
\hline & \multicolumn{2}{l}{ Arabidopsis } & & & Mouse & & Mango \\
\cline { 2 - 3 } & Real & Simulated & & Real & Simulated & Real \\
\hline Compacta & 33,542 & 21,518 & & 223,169 & 19,844 & \\
Corset & 27,080 & 26,414 & & 95,079 & 23,716 & & 38,448 \\
Grouper & 27,949 & 23,026 & & 57,501 & 18,652 & 38,063 \\
\hline
\end{tabular}

perform such analyses to obtain optimum information from the data. Each $d$ value gives $z$ clusters, and the researcher can then select $z$ contigs (one for each cluster) and use tools such as BLAST [35] to identify representative contigs in the transcriptome of a related species, or BUSCO [37] to assess contig set completeness. Test runs of differential expression programs, such as edgeR [38], can be performed with each representative set of contigs to evaluate the suitability of the results for achieving the particular aims of an RNA-Seq experiment. Here we show results obtained by running Compacta with the Arabidopsis assembly that generated the speed test results (Fig. 1; see Source data and Software evaluation and Section 3 of Additional file 1 for details).

The Arabidopsis assembly resulted in 106,895 contigs that were sent as a query to a BLAST database containing the full Arabidopsis cDNA set, which comprises 41, 671 different sequences. BLAST hits were filtered by coverture, bit score and E-value giving a total of 23,607 significant concordances. The Arabidopsis assembly was run through Compacta using a grid of $d$ values and with the parameter $l$ set to its default value of 2 (for details see Section 3 of Additional file 1).

Table 3 shows that the dynamic range of Compacta for this assembly goes from $z_{\text {min }}=13,770$ clusters when $d=0$ to $z_{\max }=103,262$ clusters when $d=1$; a 7.5 -fold change between the maximum and minimum values. By taking the largest contig as representative of each cluster, the number of distinct Arabidopsis sequences identified (column $n_{A s}$ in Table 3 ) varies from a minimum of 3344 when $d=0$ to a maximum of 23,607 for $d=1$. This latter value corresponds to the total number of Arabidopsis sequences 
Table 3 Compacta results for the Arabidopsis assembly. $d$ Parameter value, $z$ - Number of clusters (representative contigs), $n_{A s}$ - Number of Arabidopsis sequences identified

\begin{tabular}{llll}
\hline$d$ & & $z$ & $n_{\text {AS }}$ \\
\hline 0.000 & & 13,770 & 3344 \\
0.035 & $\left(d_{d}\right)$ & 28,704 & 18,381 \\
0.500 & & 34,860 & 20,455 \\
0.955 & $\left(d_{r}\right)$ & 40,656 & 21,254 \\
1.000 & & 103,262 & 23,607 \\
\hline
\end{tabular}

identified in the entire assembly. The ratio between the maximum and minimum of Arabidopsis sequences identified is $23,607 / 3344 \approx 7$, which is similar to the ratio $z_{\text {max }}$ $z_{\text {min }}$ of $\approx 7.5$ However, we will see that the proportion of changes in the number of clusters and identified sequences do not follow a linear function of $d$.

The percentages of clusters, $z$, number of identified sequences, $n_{A s}$, and clustering efficiency, $E f$, defined as $E f=$ $n_{A s} / z$, are estimated as curve functions of $d$, which again does not have a linear relationship as shown by the grey dashed line with slope 10 (Fig. 2). Nevertheless, these three curvilinear functions show a relatively small slope $(\approx$ 0 ) for $d$ values between 0.1 and 0.9 , whereas in the left and right hand extremes the three functions show sudden slope changes; from very high to relatively low near $d=$ 0.035 and, conversely, from a relatively low to a very high slope near $d=0.955$ (Fig. 2; see Table 3 for the estimated values at these critical points). Although generalizations cannot be made based on this one example, it is reasonable to assume that for almost all assemblies the values for number of clusters, identified sequences and efficiency will be non-linear functions of $d$ having two critical points at which a sudden slope change at $d_{l} \approx 0$ and $d_{r} \approx 1$ and a relatively flat change (low slope) around $d=0.5$ occur. The values for $d_{l}$ and $d_{r}$ are shown in Table 3 for the Arabidopsis assembly, and these values can be easily estimated for any particular assembly.

The curves for the Arabidopsis assembly show that there will be an unavoidable loss of accuracy in the analysis of assemblies obtained de novo from RNA-Seq experiments (Fig. 2). This inaccuracy results from not taking into account genome architecture, which in turn can confound identification of the gene from which each transcript originated to create multiple putative transcripts of which an unknown proportion could be artifacts, i.e., transcripts that do not exist physiologically. Fortunately, clustering assemblies to reduce this complexity can address the problem.

In the following discussion we will assume that the researcher has putatively identified all original contigs, has determined the approximate points $d_{b} d_{r}$ for the assembly of interest, has obtained sets of representative contigs at $d$ equal to $0, d_{b}, 0.5, d_{r}$ and 1 , and performed differential expression analyses for all $d$ values shown above. We will be looking for a 'Goldilocks' point for $d$ where there are not 'too few' or 'too many' contigs to obtain biologically relevant knowledge from our data.

Analyses of a representative set of contigs at $d=0$ will give the minimum resolution of the assembly, because all representative contigs at that point represent completely independent 'genes' or 'gene families' and consequently the number of identified sequences will be a minimum (Fig. 2; blue dotted line). Based on this selection, all reads mapping to the contigs will have a unique hit, and thus the statistical power of detection of differentially expressed entities will be a maximum, because reads are not shared between the entities analyzed. Aside from other considerations, the differential expression analysis at $d=0$ has the advantage of displaying a broad scenario; entities that are differentially expressed will show sets of genes that are surely affected by experimental conditions, even if many splicing variants and other similar transcripts are grouped and represented by a single transcript.

However, for many purposes, the number of representative sequences at $d=0$ will be 'too few' and further analyses will be needed to improve accuracy.

On the other extreme, at $d=1$, we have the largest number of representative contigs, and consequently the largest number of identified sequences (Fig. 2; dotted blue line). However, the procedure efficiency is lowest at this point (Fig. 2; dashed green line), meaning that many contigs represent the same identified sequence ( $E f=n_{A s}$ / $z$ is at its minimum), and thus very little advantage is gained from the clustering procedure, because many representative sequences will be redundant and characterize the same gene. At this point, the statistical power is also reduced because fewer reads map to each individual contig when compared with the other extreme (i.e., $d=0$ ). Thus, $d=1$ will give us 'too many' contigs.

Examining the point around which $d=d_{l}$ for the Arabidopsis assembly gives $d \approx 0.035$ (Table 3 ). At approximately this point the curves for $z, n_{A s}$ and $E f$ show a sudden slope change, going from a sharp increase to a more steady state that will continue for values of $d>d_{l}$ and up to $d<d_{r}$. The $d_{l}$ point gives large increases in the number of contigs, $z$, and identified sequences, $n_{A s}$, when compared with the point at $d=0$, say $\Delta z(d)=z\left(d=d_{l}\right)-$ $z(d=0)=28,704-13,770=14,934 \quad(\approx 15 \%$ of increment $)$, $\Delta n_{A s}(d)=n_{A s}\left(d=d_{l}\right)-n_{A s}(d=0)=18,381-3344=15,037$ $(\approx 65 \%$ of increment; see Table 3 and Fig. 2$)$. Also near $d=d_{l}$, at $d=0.05$, we obtain the maximum efficiency, $\max$ $(E f)=18,381 / 19962 \approx 0.92$ (see Fig. 2 and Section 3 of Additional file 1 for details). In the context of information content, the $\mathrm{d}$ value at which $\max (E f)$ is reached is optimal; i.e., we will not have 'too few' or 'too many' contigs to represent assembly diversity and thus this point is the 
'Goldilocks point on $d$ '. If the differential expression of the representative contigs at max $(E f)$ is satisfactory for the aims of the RNA-Seq experiment, the analysis at this point could be reported as the final result.

The pipeline sketched here to obtain an optimum point for the parameter $d$ that corresponds to the maximum efficiency, max $(E f)$, can be easily performed with an assembly obtained from any RNA-Seq experiment. In general, the most complex decision for the researcher is selection of a well-known and closest organism with which to suitably compare the organism under study. When such selection is done, a simple BLAST experiment using all contigs from the assembly as queries and the full transcriptome of the known organism as the target can be easily performed (details of this procedure are presented in Section 3 of Additional file 1 for the Arabidopsis assembly). Alternatively, or additionally, the researcher could perform a BUSCO experiment (see [35] and the website with [37]). The general lines of the procedure are, first, to obtain all BUSCO terms that correspond to all contigs in the assembly, and upon gathering these terms, use the sets of representative contigs obtained with Compacta with a grid of $d$ values. As with $B L A S T$, with BUSCO we can obtain a point $d$ that will correspond to the maximum efficiency, $\max (E f)$, but in this case the $E f$ for each value of $d$ is defined as the number of BUSCO terms found over the number of contigs. An additional advantage of the BUSCO approach is that the terms found will generally have straightforward biological interpretations, which is useful for understanding differential expression analyses.

\section{Comparing Compacta with other clustering tools}

Compacta does not directly use sequence information (as do tools like CD-HIT [15]) but instead uses reads that are shared between contigs. It also does not use statistical approaches to try to determine the gene of origin for each contig (as do Corset or Grouper), and as such is not fully comparable with all clustering tools. However, we did perform various comparisons and the results are summarized below, with details and other analyses presented in Section 2 of Additional file 1.

An ideal clustering algorithm for assemblies will correctly identify all contigs that arise from transcription of the same locus and cluster all of these contigs together. On the other hand, contigs arising from transcripts originating from different loci will always belong to different clusters. However, two kinds of errors, false positives (where two contigs from different loci are clustered together) and false negatives (where two contigs that belong to the same locus are not clustered together) can occur.

In Table 4 we present four possibilities for the classification of contig pairs, and the frequency of each one of the cases (in each of the 4 cells of the table) is represented by $a, b, c$ and $d$, whereas the sum of the frequencies, $a+b+c+d$, gives the total number of pair-wise contig comparisons. A perfect clustering algorithm will have $b=0$ and $c=0$, and we can define two metrics to measure an algorithm behavior, termed 'Recall', $R=a$ / $(a+c)$ and 'Precision', $P=a /(a+b)$. Clearly, the perfect algorithm will have $R=1$ and $P=1$, whereas $R<1$ imply the existence of false negatives $(c>0)$ and $P<1$ implies the existence of false positives $(b>0)$.

We estimated recall, $R$, and precision, $P$, of 5 clustering algorithms for Arabidopsis and mouse assemblies that were used to evaluate execution time (see Fig. 1 and Source data and Software evaluation). We again compared Compacta with mainly graph-based Corset and Grouper and also with CD-HIT, which relies exclusively on sequence information [15] and the Trinity program for contig grouping [39], which is based on De Bruijn graphs. For the comparison, all five programs were run using default parameters.

The program having the lowest recall for both assemblies was $C D-H I T$, and the values significantly differed from the higher values produced by the other 4 programs (Fig. 3). This difference could be because $C D$-HIT uses only information for direct contig likeness at the sequence level, and thus produces multiple false negatives when contigs that are truly related are not grouped. On the other hand, CD-HIT had the highest precision for both assemblies, implying a low frequency of false positives that again is attributable to the direct use of sequence information: when CD-HIT clusters two or more contigs, it does so based on high sequence similarity. A disadvantage of $C D-H I T$ is that the clusters it produces contain only a small number of contigs -those that are highly similar at the sequence level, and the user has little control over the degree of assembly compression (data not shown; see Section 2 of Additional file 1).

We also observed that the recall ratios for Corset, Compacta, Grouper and Trinity were relatively similar within both mouse and Arabidopsis assemblies, but the values for mouse were always lower than those for Arabidopsis (Fig. 3). The higher complexity of the mouse assembly relative to that for Arabidopsis can explain the generally lower recall ratios of the four programs and that higher numbers of false negatives can be produced with complex assemblies. Compacta had the highest recall for Arabidopsis, while Grouper had the highest recall for the mouse assembly.

In contrast to the results for recall, for all five programs precision was marginally higher for the mouse assembly relative to that for Arabidopsis (Fig. 3), suggesting that the relative proportion of false positives does not increase with assembly complexity. 
Table 4 Case classification for contig pairs after clustering. $a, b, c$ and $d$ are frequencies resulting from a clustering experiment

\begin{tabular}{lllll}
\hline & & & & \\
\cline { 3 - 5 } & & Yes & No locus? & Total \\
\hline Clustered? & Yes & $a$ (true positive) & $b$ (false positive) & $a+b$ (positives) \\
& No & $c$ (false negative) & $d$ (true negative) & $c+d$ (negatives) \\
& Total & $a+c$ (single locus) & $b+d$ (different loci) & $a+b+c+d$ \\
\hline
\end{tabular}

In terms of precision, and excluding the CD-HIT case discussed above, in all cases Corset, Compacta, Grouper and Trinity have a precision around 0.8 (Fig. 3); at $P=$ 0.85 and $P \approx 0.84$ Corset and Grouper, respectively, had the highest precision for the Arabidopsis assembly, whereas Compacta and Trinity both had $P \approx 0.78$. For the mouse assembly, Corset had the highest precision $(P \approx 0.95)$ while Compacta and Grouper both had $P \approx 0.9$, and Trinity had $P \approx 0.89$. Even when Compacta did not have the highest precision in the assemblies studied, it was faster than the other programs, and, importantly, can be adjusted to yield an optimum number of clusters with the highest efficiency (see previous section). These advantages compensate for the minor loss of precision upon comparing Compacta with Corset and Grouper. Furthermore, because Compacta does not try to determine the gene origin of each contig, its results are independent of the RNA-Seq experimental design (treatments), whereas Corset and Grouper are affected by experimental factors due to the use of statistical tools to try to estimate genes from which contigs originated.

We compared the precision and recall for Compacta with a yeast assembly and generated sets of simulated assemblies for Arabidopsis, mouse and yeast with plots of precision $\times$ recall for all comparisons (Section 2 in Additional file 1). We also compared differential expression patterns from various assemblies (Section 4 in Additional file 1). Together these results showed that Compacta efficiently detected differential expression patterns after assembly clustering.

\section{Conclusions}

In most cases de novo assemblies produce an excess number of contigs, many of which represent minor transcription variants from expression of the same gene. We assert that without full genome information segregating contigs by gene of origin is very difficult, if not impossible. Thus, for genomes that do not have complete information, researchers must reduce the analytical complexity by selecting a set of contigs to represent the entire transcriptome. Compacta provides flexibility in the selection of sets of representative contigs for downstream analysis. Its dynamic range goes from maximum transcriptome compression -wherein all contigs that share common reads are represented by a single contig, down to minimum compression, wherein only those contigs that are subsets of the same reads are clustered together and thus represented by a single contig. Because Compacta is fast, many test runs can be made to find the optimum level of transcriptome compactness according to the specific aim of a given experiment.

\section{Availability and requirements \\ Project name: Compacta.}

Project home Page: https:/github.com/bioCompU/ Compacta or https://doi.org/10.5281/zenodo.3469484 Home page includes a source code tar ball, compiled executables and a software manual with demos.

Operating Systems: any Unix-based system with proper installation, or Linux $\times 86$ with standalone executable.

Programming Language: $\mathrm{C}++$.

Other Requirements: Samtools, zlib library and a $\mathrm{C}++$ compiler.

Recommended Hardware: The memory needed to run the software is determined by the size of the largest input BAM file. Compacta loads the input files in memory and releases them after data pre-processing. As such, when the input data are loaded, only a small amount of memory is needed for processing. For a given dataset Compacta typically requires more memory than that of tools such as CD-HIT-EST, but less than that needed by Corset and transcriptome assemblers. The experiments shown in this study were performed on a computer with 64 Gb RAM, and an Intel CoreTM ${ }^{\mathrm{sm}}$ processor i7-6700 with 4 cores.

License: GNU GPL version 3.

Restrictions for use by non-academics: None.

\section{Supplementary information}

Supplementary information accompanies this paper at https://doi.org/10. 1186/s12864-020-6528-x.

Additional file 1. Supplementary results. Additional text and figures are ordered according to sections in the main text

Abbreviations

BAM: Binary Alignment Map; bp: base pair; cDNA: complementary DNA; Ef: Efficiency; mRNA: messenger RNA

\section{Acknowledgements}

The authors thank Juan Bautista Teran Fraijo and María del Socorro Fraijo Encinas for helping us secure financial support from COFUPRO. We also thank Oscar Julio Luna, Cecilia Piri Alcaraz, Lucia Preciado Gamez and Hugo Piri for administrative support during the project. We are grateful to Dr. 
Miguel A. Hernández-Oñate for support during the development of this software and for providing the de novo mango assembly.

\section{Authors' contributions}

FR-M programmed Compacta, performed the analyses and wrote the first draft of the manuscript. $\mathrm{CH}-\mathrm{K}$ and $\mathrm{OM}$ conceived the research, supervised both software development and analyses and wrote the final version of the manuscript. All authors reviewed and approved the final manuscript.

\section{Funding}

This work was supported in part by COFUPRO (A/GTO/RGAG-2014-076Consorcio de Fundaciones PRODUCE). FR-M acknowledges the Mexican Council of Science and Technology (CONACyT) for support from a PhD scholarship (261122) during the development of the project. The funding body did not contribute to the design of the study or collection, analysis and interpretation of data and in writing the manuscript.

\section{Availability of data and materials}

The datasets used in this study are available in the Sequence Read Archive (SRA) with accession numbers SRP043494, ERP016911 and SRP149554 for Mango, Arabidopsis and Mouse datasets, respectively. The source code and standalone executable of the version of Compacta used in this study are available at https://doi.org/10.5281/zenodo.3469484.

\section{Ethics approval and consent to participate}

Not applicable.

\section{Consent for publication}

Not applicable.

\section{Competing interests}

The authors declare that they have no competing interests.

Received: 2 October 2019 Accepted: 22 January 2020

Published online: 11 February 2020

\section{References}

1. Adams MD, Kelley JM, Gocayne JD, Dubnick M, Polymeropoulos MH, Xiao H, Merril CR, Wu A, Olde B, Moreno RF, et al. Complementary DNA sequencing: expressed sequence tags and human genome project. Science. 1991; 252(5013):1651-6

2. Ozsolak F, Milos PM. RNA sequencing: advances, challenges and opportunities. Nat Rev Genet. 2011:12(2):87.

3. Grabherr MG, Haas B, Yassour M, Levin JZ, Thompson D, Amit I, Adiconis X Fan L, Raychowdhury R, Zeng Q, Chen Z, Mauceli E, Hacohen N, Gnirke A, Rhind N, Di Palma F, Birren B, Nusbaum C, Lindblad-Toh K, Regev A. Fulllength transcriptome assembly from rna-seq data without a reference genome. Nat Biotechnol. 2011;29:644-52. https://doi.org/10.1038/nbt.1883.

4. Luo R, Liu B, Xie Y, Li Z, Huang W, Yuan J, He G, Chen Y, Pan Q, Liu Y, Tang J, Wu G, Zhang H, Shi Y, Liu Y, Lu Y, Han C, Cheung DW, Yiu S-M, Peng S, Xiaoqian Z, Liu G, Liao X, Li Y, Yang H, Wang J, Lam T-W, Wang J. SOAPdenovo2: an empirically improved memory-efficient short-read de novo assembler. GigaScience. 2012;1(1):2047. https://doi.org/10.1186/2047217X-1-18.

5. Birol I, Jackman SD, Nielsen CB, Qian JQ, Varhol R, Stazyk G, Morin RD, Zhao Y, Hirst M, Schein JE, Horsman DE, Connors JM, Gascoyne RD, Marra MA Jones SJM. De novo transcriptome assembly with ABySS. Bioinformatics. 2009:25(21):2872-7. https:/doi.org/10.1093/bioinformatics/btp367 http/// oup.prod.sis.lan/bioinformatics/article-pdf/25/21/2872/6058661/btp367.pdf

6. Bankevich A, Nurk S, Antipov D, Gurevich AA, Dvorkin M, Kulikov AS, Lesin VM, Nikolenko SI, Pham S, Prjibelski AD, Pyshkin AV, Sirotkin AV, Vyahhi N, Tesler G, Alekseyev MA, Pevzner PA. Spades: A new genome assembly algorithm and its applications to single-cell sequencing. J Comput Biol. 2012;19(5):455-77. https://doi.org/10.1089/cmb.2012.0021

7. Martin JA, Wang Z. Next-generation transcriptome assembly. Nat Rev Genet 2011;12(10):671.

8. Audano PA, Sulovari A, Graves-Lindsay TA, Cantsilieris S, Sorensen M, Welch AE, Dougherty ML, Nelson BJ, Shah A, Dutcher SK, et al. Characterizing the major structural variant alleles of the human genome. Cell. 2019;176(3): 663-75.
9. Koonin EV. Orthologs, paralogs, and evolutionary genomics. Annu Rev Genet. 2005;39:309-38.

10. Indrischek H, Wieseke N, Stadler PF, Prohaska SJ. The paralog-to-contig assignment problem: high quality gene models from fragmented assemblies. Algorithms Mol Biol. 2016;11(1):1.

11. Huang Y-Z, Passey DA, Yang Z, Yu J, Wong GK. Is "junk" dna mostly intron dna? 2015.

12. Wong GK-S, Passey DA, Yu J. Most of the human genome is transcribed. Genome Res. 2001;11(12):1975-7.

13. Ameur A, Zaghlool A, Halvardson J, Wetterbom A, Gyllensten U, Cavelier L, Feuk $L$. Total RNA sequencing reveals nascent transcription and widespread co-transcriptional splicing in the human brain. Nat Struct Mol Biol. 2011; 18(12):1435

14. Chou H-C, Acevedo-Luna N, Kuhlman JA, Schneider SQ. Pdumbase: a transcriptome database and research tool for platynereis dumerilii and early development of other metazoans. BMC Genomics. 2018;19(1):618.

15. Li W, Godzik A. Cd-hit: a fast program for clustering and comparing large sets of protein or nucleotide sequences. Bioinformatics (Oxford, England). 2006:22:1658-9. https://doi.org/10.1093/bioinformatics/btl158.

16. Davidson NM, Oshlack A. Corset: enabling differential gene expression analysis for de novo assembled transcriptomes. Genome Biol. 2014;15:410. https://doi.org/10.1186/s13059-014-0410-6.

17. Malik L, Almodaresi F, Patro R. Grouper: graph-based clustering and annotation for improved de novo transcriptome analysis. Bioinformatics. 2018;34(19):3265-72. https://doi.org/10.1093/bioinformatics/bty378.

18. Vlasova A, Capella-Gutiérrez S, Rendón-Anaya M, Hernández-Oñate M, Minoche AE, Erb I, Câmara F, Prieto-Barja P, Corvelo A, Sanseverino W, et al. Genome and transcriptome analysis of the mesoamerican common bean and the role of gene duplications in establishing tissue and temporal specialization of genes. Genome Biol. 2016;17(1):32.

19. Boley N, Stoiber MH, Booth BW, Wan KH, Hoskins RA, Bickel PJ, Celniker SE, Brown JB. Genome-guided transcript assembly by integrative analysis of RNA sequence data. Nat Biotechnol. 2014;32(4):341.

20. Bennetzen JL, Ma J, Devos KM. Mechanisms of recent genome size variation in flowering plants. Ann Bot. 2005;95(1):127-32.

21. Lee S-I, Kim N-S. Transposable elements and genome size variations in plants. Genomics Inform. 2014;12(3):87.

22. Stranger BE, Forrest MS, Dunning M, Ingle CE, Beazley $C$, Thorne N, Redon R, Bird CP, De Grassi A, Lee C, et al. Relative impact of nucleotide and copy number variation on gene expression phenotypes. Science. 2007;315(5813):848-53.

23. Zhu L, Zhang Y, Zhang W, Yang S, Chen J-Q, Tian D. Patterns of exon-intron architecture variation of genes in eukaryotic genomes. BMC Genomics. 2009:10(1):47. https://doi.org/10.1186/1471-2164-10-47.

24. Langmead B, Salzberg S. Fast gapped-read alignment with bowtie 2. Nat Methods. 2012:9:357-9. https://doi.org/10.1038/nmeth.1923.

25. Kim D, Langmead B, Salzberg S. Hisat: A fast spliced aligner with low memory requirements. Nat Methods. 2015;12:357. https://doi.org/10.1038/nmeth.3317.

26. Li H, Handsaker B, Wysoker A, Fennell T, Ruan J, Homer N, Marth G, Abecasis G, Durbin R, Subgroup GPDP. The sequence alignment/map format and samtools. Bioinformatics. 2009;25(16):2078-9. https://doi.org/10.1093/ bioinformatics/btp352.

27. Essam JW, Fisher ME. Some basic definitions in graph theory. Rev Mod Phys. 1970;42(2):271.

28. Kurita T. An efficient agglomerative clustering algorithm using a heap. Pattern Recogn. 1991;24:205-9. https:/doi.org/10.1016/0031-3203(91)90062-A.

29. Mercer TR, Dinger ME, Mattick JS. Long non-coding RNAs: insights into functions. Nat Rev Genet. 2009:10(3):155.

30. Frith MC, Pheasant M, Mattick JS. Genomics: The amazing complexity of the human transcriptome: Nature Publishing Group; 2005. Eur J Human Genet. 2005; 13:894-7. https://doi.org/10.1038/sj.ejhg.5201459. published online 22 June 2005.

31. Liu H, Ma X, Han HN, Hao YJ, Zhang XS. Atprmt5 regulates shoot regeneration through mediating histone $h 4 \mathrm{r} 3$ dimethylation on krps and pre-mrna splicing of rkp in Arabidopsis. Mol Plant. 2016;9(12):1634-164. https://doi.org/10.1016/j.molp.2016.10.010.

32. Tafolla-Arellano J, Zheng Y, Sun H, Jiao C, Ruiz May E, Hernandez M, Gonzalez Leon A, Sañudo R, Fei Z, Domozych D, KC Rose J, TiznadoHernández M. Transcriptome analysis of mango (Mangifera indica I.) fruit epidermal peel to identify putative cuticle-associated genes. Sci Rep. 2017;7: 46163. https://doi.org/10.1038/srep46163.

33. GeneBank: China Agricultural University, Raw Sequence Reads. https://www. ncbi.nlm.nih.gov/bioproject/PRJNA474181/ 
34. GeneBank: Sequence Read Archive. https://www.ncbi.n/m.nih.gov/sra/

35. Zhang Z, Schwartz S, Wagner L, Miller W. A greedy algorithm for aligning DNA sequences. J Comput Biol. 2000;7(1-2):203-14.

36. Stoer M, Wagner F. A simple min-cut algorithm. J ACM. 1997;44:585-91. https://doi.org/10.1007/BFb0049404.

37. Simão FA, Waterhouse RM, loannidis $P$, Kriventseva EV, Zdobnov EM. Busco: assessing genome assembly and annotation completeness with single-copy orthologs. Bioinformatics. 2015;31(19):3210-2.

38. Robinson MD, McCarthy DJ, Smyth GK. EdgeR: a bioconductor package for differential expression analysis of digital gene expression data. Bioinformatics. 2010;26(1):139-40.

39. Haas B, Papanicolaou A, Yassour M, Grabherr M, Blood PD, Bowden J, Brian Couger M, Eccles D, Li B, Lieber M, MacManes MD, Ott M, Orvis J, Pochet N, Strozzi F, Weeks N, Westerman R, William T, Dewey C, Regev A. De novo transcript sequence reconstruction from Rna-seq using the trinity platform for reference generation and analysis. Nat Protoc. 2013;8(8):1494. https://doi. org/10.1038/nprot.2013.084.

\section{Publisher's Note}

Springer Nature remains neutral with regard to jurisdictional claims in published maps and institutional affiliations.

Ready to submit your research? Choose BMC and benefit from:

- fast, convenient online submission

- thorough peer review by experienced researchers in your field

- rapid publication on acceptance

- support for research data, including large and complex data types

- gold Open Access which fosters wider collaboration and increased citations

- maximum visibility for your research: over $100 \mathrm{M}$ website views per year

At $\mathrm{BMC}$, research is always in progress.

Learn more biomedcentral.com/submissions 